\title{
Development of a Smart Bioink for Bioprinting Applications
}

\author{
Félix E. Montero ${ }^{1}$, Rodrigo A. Rezende ${ }^{2 *}$, Jorge V. L. da Silva ${ }^{2}$ and Marcos A. Sabino ${ }^{1 *}$ \\ ${ }^{1} B^{5}$ IDA Research Group, Chemistry Department, Universidad Simón Bolivar USB, Caracas, Venezuela, ${ }^{2} 3 D$ Technologies \\ Research Group (NT3D), Renato Archer Information Technology Center (CTI), São Paulo, Brazil
}

\section{OPEN ACCESS}

Edited by:

Bahattin Koc,

Sabanci University, Turkey

Reviewed by:

Henrique de Amorim Almeida, Polytechnic Institute of Leiria, Portugal

Wenling Zhang,

University of Alberta, Canada

${ }^{*}$ Correspondence:

Rodrigo A. Rezende

rodrigo.rezende@cti.gov.br

Marcos A. Sabino msabino@usb.ve

Specialty section:

This article was submitted to

Computer-Aided and Digital

Manufacturing Technologies,

a section of the journal

Frontiers in Mechanical Engineering

Received: 06 May 2019

Accepted: 26 August 2019

Published: 27 September 2019

Citation:

Montero FE, Rezende RA, da Silva JVL and Sabino MA (2019) Development

of a Smart Bioink for Bioprinting

Applications. Front. Mech. Eng. 5:56.

doi: 10.3389/fmech.2019.00056
Biofabrication is an incipient and fast-growing field of research that continues to develop groundbreaking innovations. Considerable progress has also been made in additive manufacturing technologies, which are used for the production of complex geometry using biomaterials, such as scaffolds and, most recently, bioink-based structures. Some of the topics related to these new breakthroughs in tissue engineering and bioprinting will be discussed. Additionally, the obtained experimental results in this work contribute to the recent advances in such sciences. Formulations of sodium alginate (NaAlg)/Poly(N-Isopropylacrylamide) (PNIPAm)/ZnSO 4 -based smart bioinks were developed and characterized. The lower critical solution temperature (LCST), viscosity, and thermal stability were determined through UV-Visible spectroscopy, a rheological analysis and a thermogravimetric analysis (TGA), respectively. Furthermore, an injectability test was performed in order to assess the influence of the extrusion process in the morphology of the bioinks like scaffolds (after lyophilization) using scanning electron microscopy SEM. According to the obtained results, the scaffolds presented a structure composed of interconnected pore systems, which were affected by the presence of the $\mathrm{Zn}^{2+}$ ion, and the extrusion process. The biocompatibility of the gels was tested through a hemolysis assay using erythrocytes and the obtained results indicate that have not cytotoxic effects. For all these reasons these formulations are candidates for the obtention of intelligent bioinks applicable for bioprinting and tissue engineering.

Keywords: gels, alginate, PNIPAm, scaffolds, smart bioinks, bioprinting, extrusion, tissue engineering

\section{INTRODUCTION}

As a new century arrived, important achievements in biomaterials and biotechnologies helped to consolidate three-dimensional (3D) bioprinting as a new field in biomedicine and tissue engineering. Nonetheless, conventional techniques are not able to manufacture structures with the expected morphological, mechanical, and biological properties for such applications yet, and the growing interest, desire, and need to successfully repair and eventually produce functional and artificial tissues and organs, which demands new approaches in biofabrication, have not been satisfied. The development of the so-called "bioinks" promises to fulfill most of the requirements for these challenges, but there is still a long road toward the final goal (Atala and Yoo, 2015; Groll et al., 2018). In this article, a variety of the characteristics and properties that bioinks must have will be featured.

Tissue engineering and regenerative medicine are focused in the regeneration of cells, tissues, and organs to restitute and improve the function of damaged or ill systems and to prevent or treat 
certain diseases. One of the most relatively recent technologies in this field are 3D scaffolds which by mimicking the biochemical stimuli of the extracellular matrix can be used for cell culture, and eventually, to produce living and functional tissues that could be implanted in the body to substitute or regenerate one of its components (Li et al., 2008). The design, development and fabrication of scaffolds are a consequence of the multidisciplinary contribution of experts from different fields, since a large number of aspects must be taken into consideration during their manufacturing, such as: topography control, superficial roughness, porosity, pore shape, and size; and even the verification of the newborn tissue to guarantee the existence of desirable and suitable biological and mechanical characteristics for the application of interest (El-Sherbiny and Yacoub, 2013).

Since biomaterials are designed to interact and coexist with living organisms, they must be biocompatible and biodegradable, especially for tissue engineering applications. During the life cycle, biomaterials must not generate counterproductive reactions (Park and Lakes, 2007), which can be controlled by carefully selecting their components.

Due to its mostly inert behavior and the ease at which it reacts with multivalent cations (such as $\mathrm{Ca}^{2+}$ or $\mathrm{Zn}^{2+}$ ) to form gels, alginate is one of the most used biopolymers in cell and tissue culture (Atala and Yoo, 2015). However, it generally has a high content of contaminants and it must be properly purified before used (Dusseault et al., 2006).

Smart polymers and gels have also been widely used in biomaterials owing to their ability to reversibly respond to several chemical and physical stimuli, such as changes in temperature, $\mathrm{pH}$, luminous intensity, and electric or magnetic fields. Most particularly, according to the relative position of the thermodynamic state from thresholds known as LCST and UCST (lower and upper critical solution temperature, respectively) which establish a phase equilibrium in solution, thermosensible polymers will tend to interact more or less with molecules of their own species or with molecules of the solvent, which leads to a temperature-dependent shift in their solubility. PNIPAm is a synthetic thermosensible polymer with a LCST close to the human body temperature in aqueous solutions (around 32$34^{\circ} \mathrm{C}$ ), which makes of it an interesting candidate for applications in tissue engineering (Coronado et al., 2010; Castro et al., 2012; Carrero et al., 2018).

\section{BIOPRINTING}

3D Bioprinting arose as a flexible technique within a wide range of applications in regenerative medicine. This recent field of biotechnology can be described as additive manufacturing or layer by layer construction of patterns and structures where cells are deposited by applying 3D printing technologies under conditions that guarantee their viability and biologic development in a way that allows the creation of tissues and organ-like structures (Sabino et al., 2017). By using a 3D printing computer-controlled device, different types of cells and biomaterials can be precisely deposited in previously designed high resolution geometries that resemble the tissue intended to reconstruct, in order to develop anatomically correct biologic structures or to create more complex structures (Rezende et al., 2015).

Just as a conventional office printer requires of ink cartridges to print on a sheet of paper, a bioprinter requires of "bioink," a cell-laden comprised viscous fluid, hydrogel, or polymeric solution (sometimes without any cell load) that gives the mechanical support and the ideal environment for the cell culture. The main long-term goal of bioprinting technologies is to build the first fully-functional organ. This have not been achieved yet, however, some scientists have claimed to successfully obtain low scale organoids that have been implemented in several applications, such as pathologic and toxicologic testing and drug development (Atala and Yoo, 2015). Furthermore, 3D bioprinting has been proved to be a suitable technique for the obtention of scaffolds, which can be used in the regeneration of different human anatomies, such as joints and ligaments. The first $3 \mathrm{D}$ bioprinting registered patent was presented in USA back in 2003, and granted in 2006, which makes of this a novel technology (Mironov et al., 2008; Sabino et al., 2017).

$4 \mathrm{D}$ Printing is a recently developed approach that promises to represent an important shift in additive manufacturing due to its ability of transform over time by implementing multi-material prints, or to change from one shape to another right off the print bed by using customized-material systems (Raviv et al., 2014). Several activation methods, such as heat, microwaves, $\mathrm{pH}$, temperature and light can be utilized to start up a $4 \mathrm{D}$ printing process.

$4 \mathrm{D}$ printing was envisioned as a combination of insights on both material programmability, and design/engineering aspects. The physics of $4 \mathrm{D}$ printing often require multiple materials to be embedded into a single $3 \mathrm{D}$ structure, thus multi-material-compatible printing techniques and meticulous planning for accurate transformations are vital requirements for such technology (Raviv et al., 2014; Li et al., 2017).

By applying stimuli-responsive materials and advanced 3D bioprinting strategies, $4 \mathrm{D}$ bioprinting has been leveraged to create dynamic 3D-patterned biological structures that can transform their shapes or behavior under various stimuli (Li et al., 2017). Thus, it is easy to verify that the biomaterials studied in this paper can be of interest for $4 \mathrm{D}$ bioprinting.

\section{BIOPRINTERS AND BIOINKS}

The need to fabricate tissues and organs and the rise of micro and nanometric 3D printing technologies have resulted in the development of 3D scaffolds of spatially controlled patterns that can be conditioned biologically to direct the purpose of cells. Even though bioprinting is still considered to be in its early stages, it continues to gain popularity among regenerative medicine researchers due to its huge potential (Atala and Yoo, 2015).

An important aspect that is usually considered as a bottleneck for the design and implementation of bioprinting systems is the conception of the bioink, that is, "a formulation of cells suitable for processing by an automated biofabrication technology that may also contain biologically active components 
and biomaterials" (Groll et al., 2018). They are typically made up of cellular material, additives (growth factors, signaling molecules, etc.), and a supportive scaffold (mostly polymers). An ideal bioink should possess proper mechanical, rheological, and biological properties of the target tissues, which are essential to ensure correct functionality of the bioprinted tissues and organs (Gungor-Ozkerim et al., 2018).

The selection of the materials used for bioink scaffolds tends to deviate more from the selection of those used in traditional tissue engineering strategies, such as main bioink components, cellular materials and additive factors. This is mainly because suitable scaffold properties are crucial to support the physical demands of the printing process. Bioink scaffolds must provide cells with secure attachment and protection from the mechanical and thermal stresses of printing (Wang et al., 2015; Bishop et al., 2017). They also must support cellular growth and proliferation without affecting the cell phenotype (Markstedt et al., 2018; Yin et al., 2018). Adequate biocompatibility is the greatest limiting factor for potential scaffolds, as they must be cytocompatible and must not cause any immune or inflammatory response, or premature stem cell differentiation. The diversity of specific bioink scaffolds used by researchers is extensive, and those scaffolds should present a biomimetic morphology (extracellular matrix) (Pati et al., 2016; Derakhshanfar et al., 2018).

By controlling the physical and chemical properties of the extracellular matrix (ECM), the behavior of the cells can be regulated to accelerate the integration of the tissue or the functional recovery and to improve its temporal and mechanical performance. That is why it is fundamental to carefully choose the polymers to be used in the formulations (Atala and Yoo, 2015).

Many scientists use natural polymers as the main component of 3D scaffolds, since, even though they lack mechanical stability, they are mostly water-soluble under physiological conditions and resemble the extracellular matrix both chemically and structurally. This allows them to mimic the native microenvironment of the cells, thus improving and modulating the adhesion, migration, proliferation, and differentiation process; which is why around $90 \%$ of the polymers used in bioprinting are from natural origin. The most common natural polymers used in bioprinting are collagen, alginate, hyaluronic acid, and gelatin, as shown in Figure 1: an adaptation of (Atala and Yoo, 2015) about a distribution of natural and synthetic polymers employed on bioinks based on a compilation from relevant literature. On the other hand, synthetic polymers are mostly soluble in toxic solvents or have a melting points above the human body temperature, which compromises cell viability and makes in situ cell encapsulation a difficult process, which is the reason that they only occupy about $10 \%$ of the polymers used in bioprinting, being polyethylene glycol (PEG), poly(lacticco-glycolic) acid (PLGA), poly(e-caprolactone) (PCL), and poly(L-lactic) acid (PLA) the most commonly used. However, they can be easily adapted to fulfill specific properties, such as optimized mechanical properties and degradation rate, and can be functionalized with a wide range of bioactive factors.

A mixture between natural and synthetic polymers may result in a probable optimized formulation with benefits from both

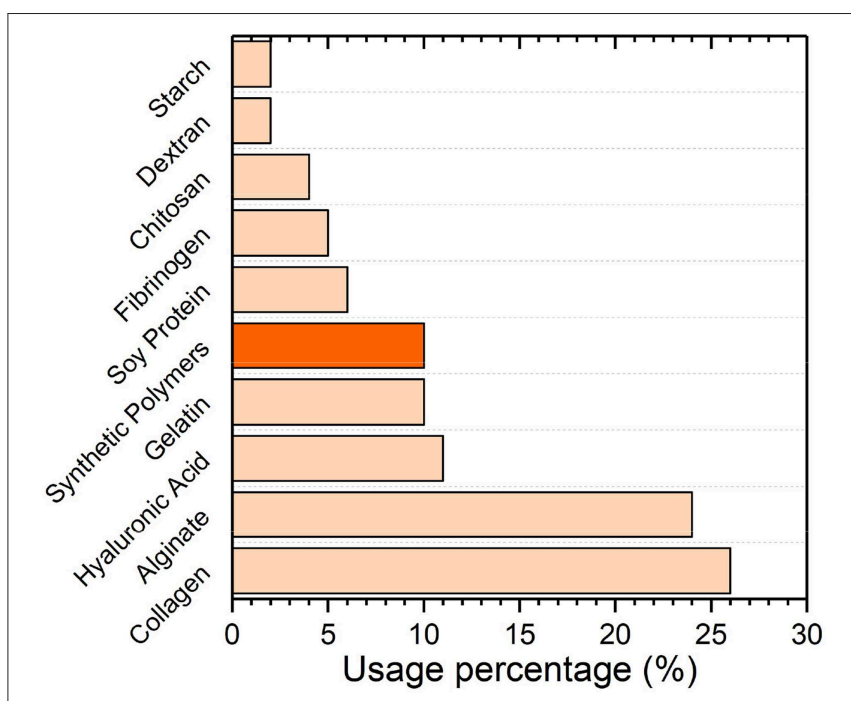

FIGURE 1 | Most used polymers in bioprinting-related researches.

materials, which can help to improve and customize the cellular response inside the 3D scaffold, thus improving the efficiency of the regeneration process. For instance, synthetic polymers can be copolymerized with biopolymers to incorporate enzymatic degradation sites and improve cell migration in the scaffold (Atala and Yoo, 2015) or even to achieve a water-soluble product.

The concerning bioprinting processes and their advantages and disadvantages are summarized in Figure 2 and Table 1, respectively (Mironov et al., 2008; Wang et al., 2015; Bishop et al., 2017; Cornelissen et al., 2017; Sabino et al., 2017; Derakhshanfar et al., 2018).

Inkjet bioprinters (Figure 2A) were the first attempts in bioprinting by using a commercial $2 \mathrm{D}$ inkjet device modified to print biological ink by layers (Wang et al., 2015; Bishop et al., 2017; Cornelissen et al., 2017). They operate under the drop-ondemand contact technique that may use thermal, piezoelectric or electromagnetic forces to expel successive drops of bioink onto a substrate to replicate a computer-aided design (CAD) with printed tissue. Although there were worries that the inkjet printing approach would harm cell viability due to the very high local temperatures in the nozzle, many studies have demonstrated that those temperature increases do not significantly impact the stability or viability of cells.

Microextrusion bioprinters (Figure 2B) produce continuous streams of bioink in contrast to the discrete droplets of inkjet bioprinters. These extremely small streams are controlled by CAD software. Unlike inkjet bioprinters, microextrusion bioprinters can successfully print high-viscosity bioinks, such as complex polymer-based, cell spheroids and clay-based substrates (Mironov et al., 2008; Bishop et al., 2017; Cornelissen et al., 2017). It is the most common bioprinting method in use nowadays. Some important data for this method are (Nair et al., 2009; Atala and Yoo, 2015): (a) extrusion viscosity $\mu<1 \times 10^{5} \mathrm{cP}$ and the ideal range of viscosity should be $300 \mathrm{cP}<\mu<0.4 \times 10^{5} \mathrm{cP}$; (b) the extrusion pressure must be $P>5 \mathrm{KPa}$, however, if $P>35$ 


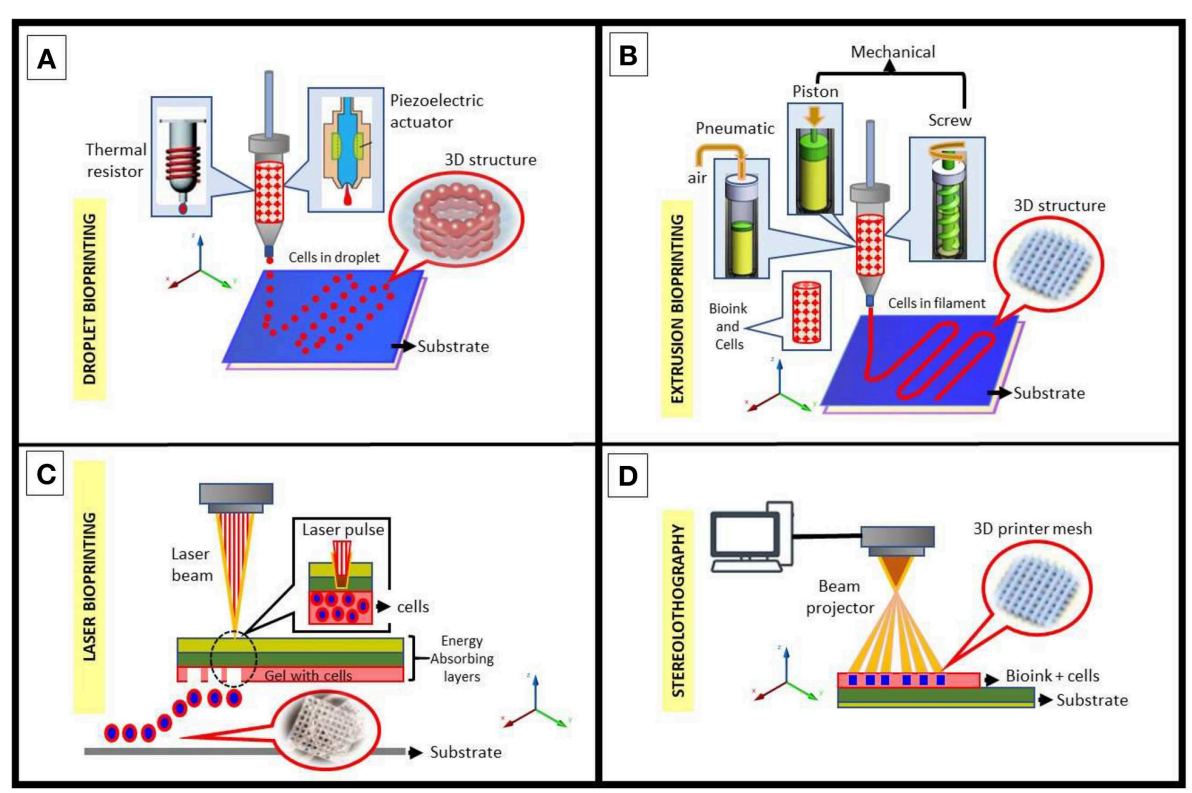

FIGURE 2 | Schematic diagram of common bioprinting methods using bioinks (with and/or without cells). (A) Inkjet printing methods: thermal and piezoelectric; both methods drive droplets through the nozzle for the construction of a 3D structure. (B) Extrusion based methods: pneumatic, piston-driven, screw-driven; these methods guarantee a continuous flow of bioink through the nozzle for the construction of a 3D structure. (C) Laser-assisted bioprinting, a nozzle-free technique consisting of a pulse laser source that deposits micro-droplets of bioink on a substrate for the construction of a 3D structure. (D) Stereolithography using a beam projector where focused light beams allow for precise photopolymerization of layers of light-sensitive polymers to apply any desired pattern to bioink.

TABLE 1 | Comparative advantages and disadvantages in Bioprinting processes.

Bioprinting methods

Inkjet

Microextrusion

Laser-assisted

Stereolithography

\section{Advantages and disadvantages}

Key benefits include high speed, availability, and relatively low costs. Disadvantages include lack of precision in regard to droplet size and placement compared to other bioprinting methods. There is also a requirement for low viscosity bioink. Frequent nozzle clogging and cellular distortion have been reported with this technique.

The major advantage of this method is the ability to print high cell densities for tissue formation. One of the major disadvantages of microextrusion bioprinting could be the distortion of cellular structure and loss of cellular viability that results from the pressure required to expel the bioink

The main advantages are the high degree precision and resolution possible for the printed structures (like micropatterned peptides, DNA, and cell arrays). Resolution as high as one cell per droplet has been achieved. Just like extrusion microprinting, laser bioprinting is able to print a high density of cells, however, one of its main limitations is a lower cell viability compared to other bioprinting methods. Another drawback is the long time required to prepare the substrate before micro-droplets of the bioink are deposited onto it.

Its main advantage is its high accuracy. But the main drawback for biomedical purposes is the intense ultraviolet radiation required to induce cross-linking processes. Other limitations are the time-consuming post-processing and the narrow range of available materials compatible for use with SLA. It is also is unable to print multiple layers. Since the polymerization process occurs in situ, one of the main drawbacks of this technique is the presence of monomer and additives leftovers, which can freely interact with the cells and compromise their viability.
$\mathrm{KPa}$, rapid and unstable extrusion could appear and compromise the quality of extrusion, thus the range of $15 \mathrm{KPa}<P<30$ $\mathrm{KPa}$ is considered as suitable for extrusion; and finally (c) the acceptable range of diameter of extruded bioink fibers must be around 5-20 $\mathrm{mm}$.

Laser-assisted 3D bioprinting (Figure 2C) is a non-contact process, nozzle-free printing process initially developed for highresolution patterning of metals, since it is often used on computer chip fabrication (Sabino et al., 2017; Derakhshanfar et al., 2018). The technology directs laser pulses through a "ribbon" containing bioink. The ribbon is supported by a titanium or gold layer capable of absorbing and subsequently transferring energy to the ribbon. The bioink and cells are suspended on the bottom of the ribbon and when vaporized by the laser pulse, create a highpressure bubble that eventually propels discrete droplets to the receiving substrate that lies just beyond the ribbon. This step is repeatedly performed to functionally create the $3 \mathrm{D}$ structures.

Finally, the stereolithography (SLA) (Figure 2D) is a bioprinting method based on photopolymerization, a process in which a UV light or laser is directed in a defined pattern over a 
path of photopolymerizable liquid monomer, thus cross-linking the polymers into a hardened layer (Sabino et al., 2017). As each layer is polymerized, the printing platform can be lowered further into the polymer solution allowing for multiple cycles to create a $3 \mathrm{D}$ structure. This technique is particularly useful when curable acrylics and epoxies are used as the photopolymerizable material (Wang et al., 2015). These substances result in a high degree of fabrication accuracy compared to other techniques.

There are different categories of bioinks according to Gungor-Ozkerim et al. (2018) as such as (a) hydrogel-based bioinks (which include protein-based bioinks, polysaccharides, synthetic polymer-based bioinks, and commercial bioinks); (b) cell aggregate/pellet-based bioinks; (c) composite bioinks/bioinks with bioactive molecules. The biofabrication of different tissue types, such as liver, vascular, cartilage, skin, bone, aortic valve, and lung tissue; has been pursued and through the use of a wide variety of bioprinting processes and cell types.

\section{NOVELTIES: DEVELOPMENT OF NAALG/PNIPAM-BASED SCAFFOLDS AND SMART BIOINKS}

Due to the importance and the wide potential of scaffolds and bioinks in biofabrication and tissue engineering, it was purposed as the main objective of this investigation to design and characterize a formulation based on alginate gels and PNIPAm as a smart polymer to develop a bioink and several structures with applications in tissue engineering. NaAlg was used as a matrix for the development of these bioinks. Since thermal sensitivity at body temperature was a desired feature for the bioinks, the smart polymer PNIPAm was selected during the design process. Just as expected, some of the results revealed a phase transition as thermosensible response of the bioinks, thus arousing an interest for the considered range of applications. In each of the following sections, the experimental procedure of this research will be explained and the obtained results will be showed.

\section{Bioink Purposed Formulations}

Sodium alginate (NaAlg) was considered as the main component of the formulation intended to use to the development of the bioink. PNIPAm was added as a disperse phase to obtain a polymer mixture with a smart behavior. The polymeric content selected for the bioinks was $5 \% \mathrm{w} / \mathrm{v}$. The first set of bioinks (F1F3) was designed by adding the synthetic polymer progressively to a control formulation of pure alginate. Additionally, the incorporation of a divalent cation was also considered to induce the formation of alginate gels. A new set of formulations (F4F6) was conceived by keeping the original NaAlg/PNIPAm proportions and adding $\mathrm{ZnSO}_{4}$ until a concentration of $0,025 \mathrm{M}$ has been reached. Nanopure water was used as a solvent for every formulation in order to avoid the presence of undesired multivalent cations in solution. Also, the NaAlg was purified according to the procedure suggested by Correa and Sabino (2018) in order to remove low molecular weight chains and any substance that may compromise the biocompatibility of the formulations. After all the components have been dissolved,

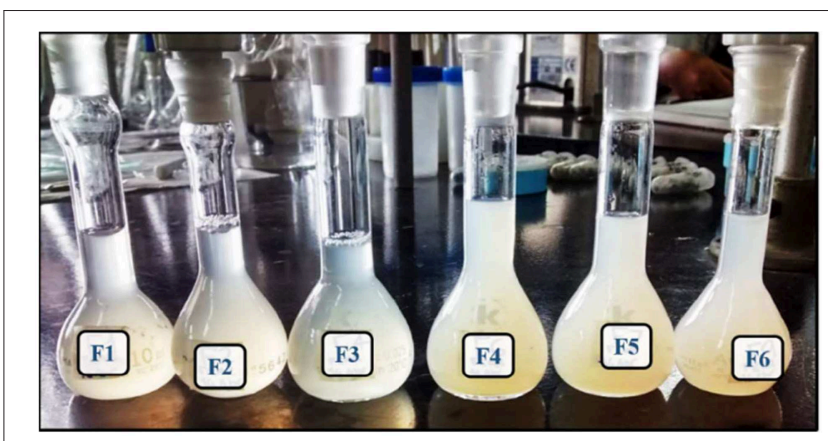

FIGURE 3 | Bioink formulations appearance at $25^{\circ} \mathrm{C}$.

TABLE 2 | Sodium alginate-based bioink formulations.

\begin{tabular}{lccc}
\hline Formulation & NaAlg $[\% w / v]$ & PNIPAm [\%w/v] & $\mathbf{Z n S O}_{\mathbf{4}}[\mathbf{M}]$ \\
\hline F1 & 5.0 & - & - \\
F2 & 4.5 & 0.5 & - \\
F3 & 4.0 & 1.0 & - \\
F4 & 5.0 & - & 0.025 \\
F5 & 4.5 & 0.5 & 0.025 \\
F6 & 4.0 & 1.0 & 0.025 \\
\hline
\end{tabular}

the bioinks, which appearance can be seen in the Figure 3, were stored in the refrigerator at a temperature of $4^{\circ} \mathrm{C}$. The composition of each formulation is listed in the Table 2.

\section{LCST Determination Through UV-Vis Spectroscopy}

Due to the presence of a dispersed phase of PNIPAm and the high proportion of NaAlg in the formulations, it was necessary to determine if the amount of the smart polymer was enough to confer its thermosensible behavior. The lower critical solution temperature (LCST) of the bioinks was determined in order to verify if they could be useful for tissue engineering purposes. This was achieved by diluting the formulations which contained PNIPAm to a concentration of $0.5 \% \mathrm{w} / \mathrm{v}$ and registering the transmittance measured by a HP AGILENT model 8452 UVVis spectrophotometer at a wavelength of $450 \mathrm{~nm}$ from 25 to $45^{\circ} \mathrm{C}$. A $0.5 \% \mathrm{w} / \mathrm{v}$ of PNIPAm was also analyzed as a control formulation. The LCST of the formulations was determined by locating the value of the temperature where the mean value of the transmittance in the negative-slope section, as shown in the Figure 4. The calculated LCST were $(32 \pm 1)^{\circ} \mathrm{C}$ for the PNIPAm solution, $(35 \pm 1){ }^{\circ} \mathrm{C}$ for the formulations F2-F3 and $(34 \pm 1)^{\circ} \mathrm{C}$ for the formulations F5-F6. The formulations F1 and F4 are not shown, since they do not content PNIPAm.

\section{Rheological Study}

The dynamic viscosity of the formulations F1, F4, F5, and F6 was determined to verify if they were suitable for the extrusion bioprinting technique, based on the criterion $\mathrm{He}$ et al. mentioned in their research in 2016. A $(10.0 \pm 0.1) \mathrm{mL}$ sample of each 


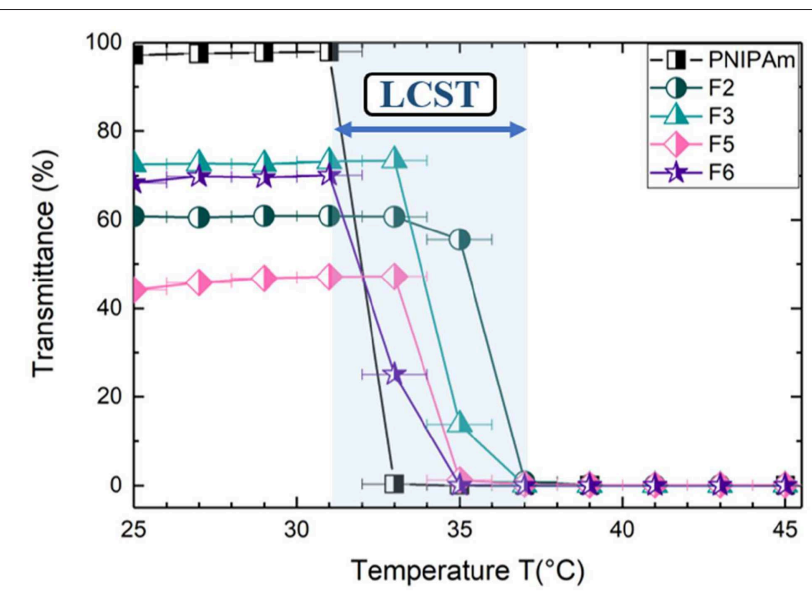

FIGURE 4 | Transmitance variation as a temperature function. LCST identification.

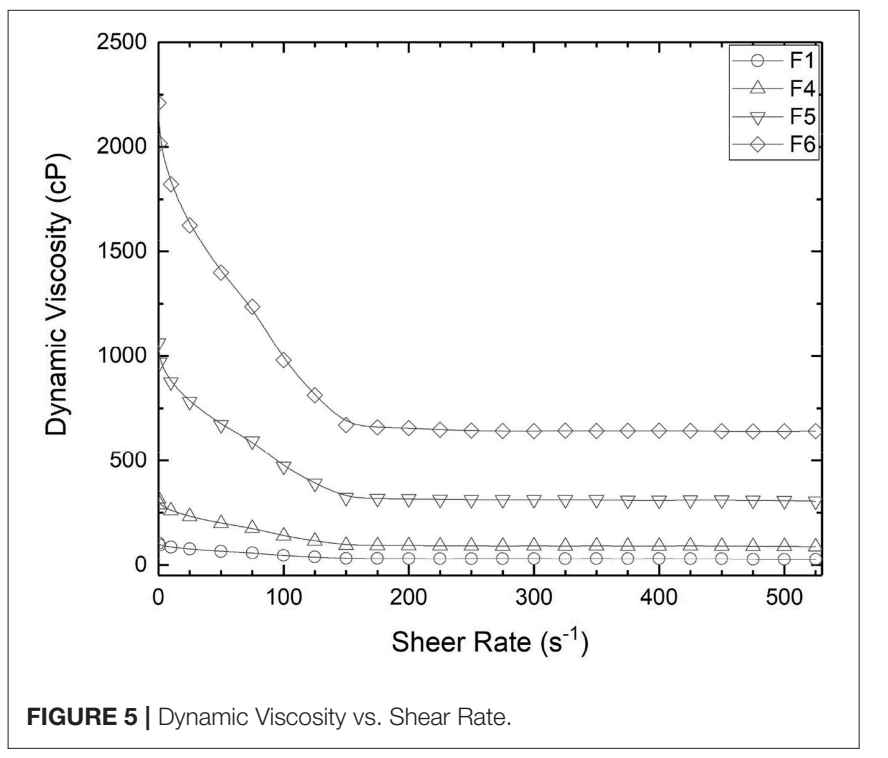

formulation was properly sealed. A Haake Mars III rheometer equipped with parallel plates with a separation of $(0.500 \pm 0.001)$ $\mathrm{mm}$ under a rotatory frequency from 0.1 to $525 \mathrm{~s}^{-1}$ at $23^{\circ} \mathrm{C}$ was used to analyze the samples. The obtained results are shown in the Figure 5. The results obtained for the formulations F2 and F3 were not presented, since their behavior was similar to that of the formulation $\mathrm{F}$ 1, however, the difference between them became significant with the addition of $\mathrm{ZnSO}_{4}$.

\section{Morphology Study: Lyophilization and Relationship With the Extrusion Process}

It is known that, because of contraction/relaxation of polymeric chains or compatibility differences between certain components, some polymeric solutions undergo structural changes when they circulate through a narrow conduct. Considering that the bioinks are generally extruded in several bioprinting techniques, a simple test was designed to assess the effect this process could have on them. $1.0 \mathrm{~mL}$ of each formulation (F1-F6) was loaded into a syringe with a stainless-steel nozzle. For the first experimental set, the bioinks were unloaded into separate Eppendorf tubes once (1X) with a syringe. For the second experimental set, after the bioinks were unloaded into separate Eppendorf tubes for the first time, the syringe was reloaded and released in each tube two more times, for a total of three discharges (3X). The samples were immediately lyophilized, fractured cryogenically, metallized and then analyzed through a JEOL model JSM6390 scanning electron microscope with a $25-\mathrm{kV}$ voltage. The most representative obtained results and photographs of the lyophilized solids are shown in the Figure 6.

\section{Thermogravimetric Analysis}

New biomaterials must undergo a sterilization processes, since it is an important requirement for biomedical applications. The thermal resistance is one of the factors that determines which type of treatment can be used for that effect. With this in mind, as a mean to determine the thermal stability of the bioinks, a thermogravimetric study was carried out. Samples of 5-20 mg from the solids obtained through lyophilization of each formulation were processed by a Mettler Toledo model TGA/SDTA851 thermogravimetric analyzer, where a temperature scanning from 25 to $600^{\circ} \mathrm{C}$ was performed at a rate of $20^{\circ} \mathrm{C} / \mathrm{min}$ under a constant flowrate of $40 \mathrm{~mL} / \mathrm{min}$ of nitrogen gas. The obtained results are shown in the Figure 7.

\section{Hemolysis Test}

Any formulation to be used as a bioink must not induce a cytotoxic response. In order to assess if the bioinks could be used in tissue engineering applications, the lysis percentage of human erythrocytes when exposed to the developed formulations was determined. The erythrocytes solution was obtained as it follows: a $25 \mathrm{~mL}$ anonymous donation of human blood was centrifuged at a speed of 2,000 rpm for $10 \mathrm{~min}$, which resulted in a biphasic solution with a yellow-colored upper layer (plasm) and a red-colored lower layer (erythrocytes). A micropipette was used to carefully remove and discard the plasm. Then, the remaining precipitate was washed with a physiological solution and centrifuged at 2,000 $\mathrm{rpm}$ for $5 \mathrm{~min}$ three times. The red precipitate obtained at the end of the third washing would be the $100 \% \mathrm{v} / \mathrm{v}$ erythrocytes solution. Then, a $2.5 \% \mathrm{v} / \mathrm{v}$ solution of erythrocytes in phosphate buffer saline (PBS) was prepared.

For the next part of the assay, $1 \mathrm{~mL} 2.0 \% \mathrm{v} / \mathrm{v}$ dissolutions of erythrocytes were prepared by adding an aliquot of $800 \mu \mathrm{L}$ of the $2.5 \% \mathrm{v} / \mathrm{v}$ erythrocytes suspension into previously identified and sterilized Eppendorf tubes, with an additional concentration of 75,150 y $300 \mu \mathrm{g} / \mathrm{mL}$ of the test agents diluted in PBS. The dissolutions for the three concentrations of each test agent (PNIPAm, $\mathrm{ZnSO}_{4}$, and the formulations F1-F6) were prepared by triplicate, for a total of 72 samples. Additionally, the negative and positive lysis controls were prepared by mixing the $800 \mu \mathrm{L}$ aliquot of the $2.5 \% \mathrm{v} / \mathrm{v}$ erythrocytes suspension with $200 \mu \mathrm{L}$ of PBS and $200 \mu \mathrm{L}$ of $1 \% \mathrm{v} / \mathrm{v}$ Triton X-100 surfactant in PBS, respectively, each by triplicate.

The 78 samples were introduced in a laboratory oven at $37^{\circ} \mathrm{C}$ for $45 \mathrm{~min}$ and then centrifuged at 2,000 rpm for $5 \mathrm{~min}$. 


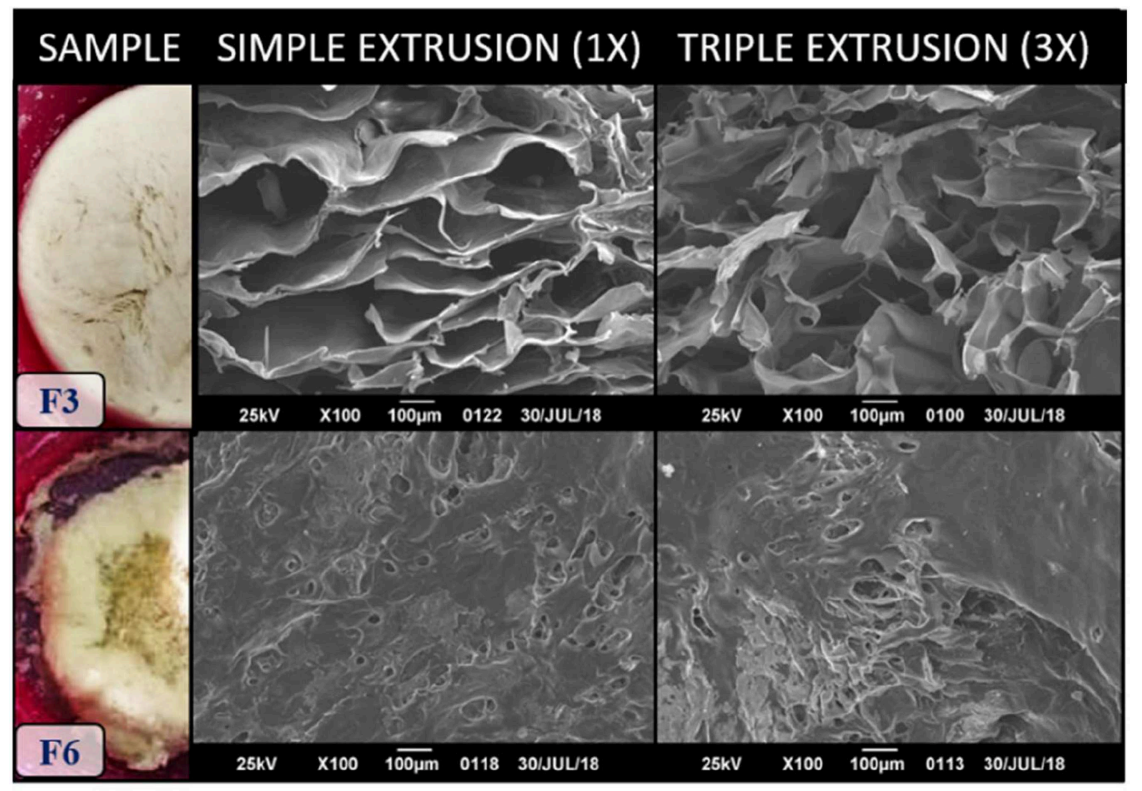

FIGURE 6 | Scanning electron micrographies comparison of the bioinks after simple $(1 X)$ and triple $(3 X)$ extrusion. The appearance of the lyophilized samples is shown on the left side.

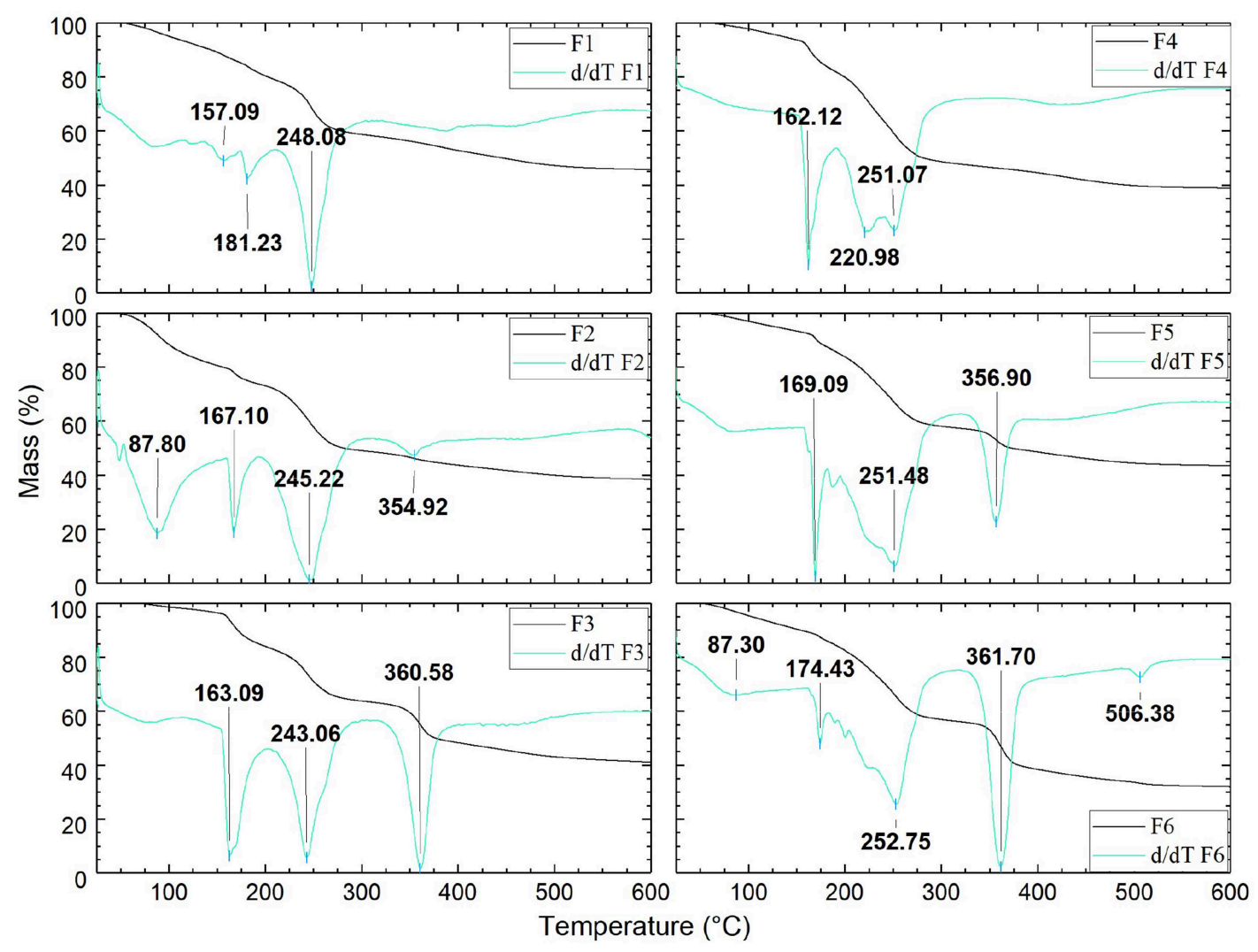

FIGURE 7 | Thermogravimetric Graphs: Formulations F1-F6. 


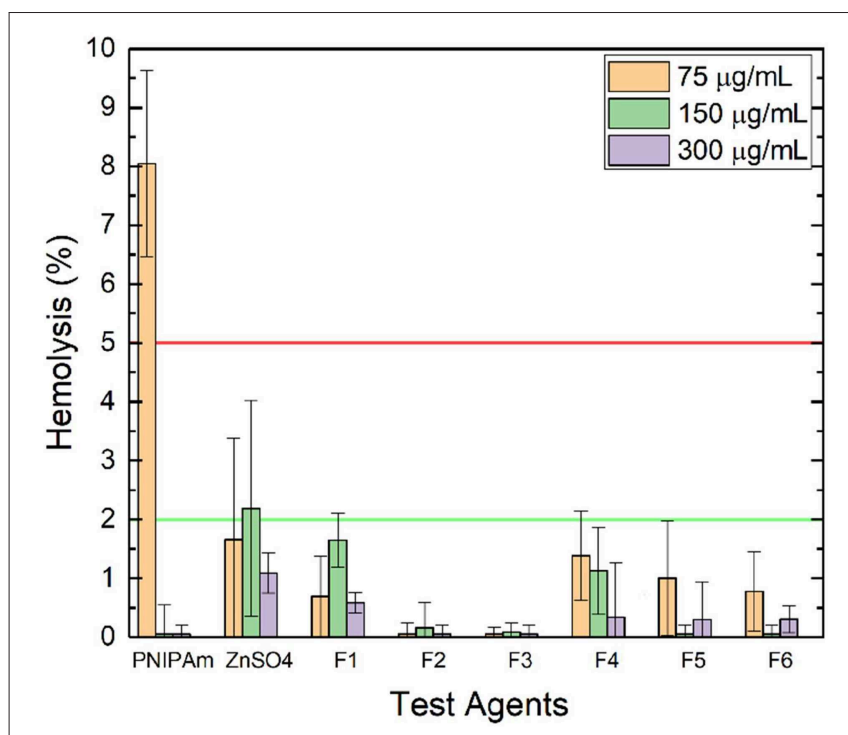

FIGURE 8 | Hemolysis percentage at 75, 150, and $300 \mu \mathrm{g} / \mathrm{mL}$.

A UV-Visible spectrophotometer HP AGILENT model 8452 was used to analyze the samples at fixed wavelength of $540 \mathrm{~nm}$ [which belongs to the hemoglobin electronic transition (Evans et al., 2013)]. The average absorbance of each test agent at each concentration was compared to those of the positive and negative controls and the lysis percentage was determined. The obtained results are shown in the Figure 8 (ASTM International, 2017).

\section{DISCUSSION}

This section focused in the characterization of the bioink and the identification of opportunities to optimize or improve its quality. The results obtained are important to assess whether the formulations can be used for the applications they're intended or not.

\section{Bioink Purposed Formulations}

A fundamental part of the bioink design is choosing the proper components according to the desired characteristics of the product. In this case, NaAlg was chosen as the base of each formulation, due to its similarity to the extracellular matrix and its improved biocompatibility, viscosity and the fact that it easily forms gels at room temperature when multivalent cations are present in solution. One of the downsides of this polymer is the lack of cellular adhesion sites, which can cause apoptosis (Atala and Yoo, 2015), and its tendency to mechanically weaken on aqueous solutions due to its water affinity and the diffusion of the cations that cause the reticulation. Both problems could be solved by adding another polymer to the formulations, such as gelatin, to increase the number of active sites (Atala and Yoo, 2015), or a synthetic polymer, to increase the mechanical stability of the gels.

During the design process, the PNIPAm arose as a candidate for the bioink due to its thermosensible properties. The phase transition that this polymer undergoes at the human body temperature in aqueous solutions can help to improve the mechanical stability of the bioink at the incubation temperature, while at low temperatures it dissolves and recovers its homogeneous solution characteristics, which will be discussed in the following sections.

In order to induce the ionic reticulation of the alginate phase, $\mathrm{ZnSO}_{4}$ was added to the formulations. This way, both the viscosity and the mechanical stability of the bioink would increase. The $\mathrm{Zn}^{2+}$ ion is considered a relatively atoxic agent so it shouldn't create any counterproductive reactions that may compromise the cell viability, however, it's important to choose a suitable concentration for that mean. The molar concentration of $0.025 \mathrm{M}$ was selected according to the research done by SerenoTocuyo on 2015, which proved not to inflict any cytotoxic effect. The formulations containing the $\mathrm{Zn}^{2+}$ ion (F4-F6) showed a higher viscosity and opacity than their analogs (F1-F3), as shown in Figure 2, which is attributed to the ionic reticulation that could promote the polymer-polymer interactions of the alginate and causes its molecular chains to achieve an organized structure, thus increasing the density of the solution.

\section{LCST Determination Through UV-Vis Spectroscopy}

The transition temperature of PNIPAm is practically independent of its concentration or its molecular weight (Gandhi et al., 2015), and this phenomenon allowed to determine its LCST from its dissolutions (Lemanowicz et al., 2014).

When the temperature of aqueous PNIPAm solutions is lower than the LCST, the hydrophilic interactions prevail (solventpolymer), the polymer stays solvated and the mixture behaves as a single homogeneous and transparent phase. When the temperature increases over the LCST, the prevailing interactions shift to hydrophobic (polymer-polymer), which plummets the PNIPAm solubility in water and turns the mixture to a white and opaque solution (Coronado et al., 2010; Gandhi et al., 2015), as shown in Figure 9.

The reflected electromagnetic radiation by the aqueous PNIPAm solution at high temperatures belongs to the visible spectrum, so it's perceived by the human eye. This peak in absorptivity at $450 \mathrm{~nm}$ can be used to determine the exact temperature at which the shift occurs through UVVis spectroscopy.

The results from Figure 4 show how the transmittance at $450 \mathrm{~nm}$ plummets due to the phase transition of PNIPAm, even in the presence of the alginate matrix. The most drastic change corresponds to the pure PNIPAm solution, whereas the other formulations have other components that possibly absorb some of the electromagnetic radiation, thus reducing the transmittance at low temperatures.

The obtained results reveal that the addition of alginate shifted the LCST to temperatures closer to that of the human body, which positions the developed formulations as possible candidates for applications in tissue engineering such as cell encapsulation, which results very promising for bioprinting applications. The effect of the $\mathrm{ZnSO}_{4}$ couldn't be determined since the LCST variation of the corresponding formulations belongs to the error margin of the measurements. 


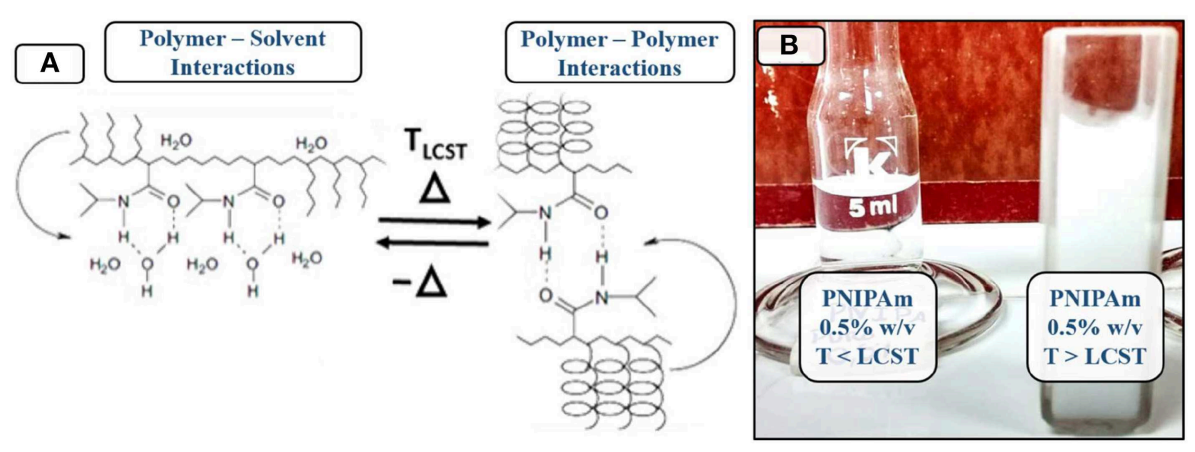

FIGURE 9 | Relationship between temperature and the phase transition of PNIPAm. (A) Molecular interaction shifts due to changes in temperature. (B) Appearance of PNIPAm solutions over and under the LCST.

\section{Rheological Study}

Viscosity can be greatly affected by parameters such as temperature, pressure, sheer rate, molar concentration, and type of fluid. For this assay, the effect of sheer rate in viscosity was determined. This is useful to determine the fluid dynamic behavior of the bioinks. Sheer rates occur then a fluid is physically displaced or distributed, as it occurs when mixing, pouring, spreading, or spraying (Janna and Simpson, 2008).

Isaac Newton was one of the first scientists to study the internal frictions of fluids: he assumed that the viscosity of all materials was independent of the sheer rate at a given temperature (Newtonian fluids). Nowadays it is known that this seems to be not a rule, but an exception, since the viscosity of most substances actually depends on the sheer rate. NonNewtonian fluids are classified as pseudoplastics of dilatants if their viscosity decreases or increases with sheer rate, respectively (Janna and Simpson, 2008). The distinction of these terms is important for bioprinting, since one wouldn't expect to develop a bioink which viscosity increased when pressure was exerted, and this behavior would actually cause a malfunction of the printing device.

According to the obtained results (Figure 4), the viscosity of all the samples is inversely proportional to the sheer rate, so they can be considered as pseudoplastic fluids. The viscosity has a maximum value when the sheer rate is minimum, however, when it reaches $150 \mathrm{~s}^{-1}$, the bioinks start to behave as Newtonian fluids, which becomes apparent as the slope of the curve tends to zero at higher values. He et al. (2016) did a research about extrusion bioprinting, where they stated that an optimal performance was achieved when the viscosity of the bioinks stayed in the range of 300 to $30.000 \mathrm{cP}$, in order to avoid smearing or clogging. According to that criterion, the formulations F5 and F6 could be used as bioinks for the extrusion technique.

\section{Morphology Study: Lyophilization and Relationship With the Extrusion Process}

Some thermosensible formulations can be used as tissue filling and drug or biomolecule carriers (which can be useful for bioink development). In order to determine the range of applications of the bioinks, it is important to verify if the extrusion process can produce changes in their morphologies (Coronado et al., 2010; Vieira et al., 2014).

For this section, the formulations will be referred as F\#$1 \mathrm{X}$ and F\#-3X, where \# symbolizes the formulation ID, in relation to the number of times they underwent the extrusion process. According to the results shown in Figure 5, the obtained structures can be considered as scaffolds, since they consist of systems of uniform pore size (Sabino et al., 2017), where the mean size is affected by the presence of the $\mathrm{Zn}^{2+}$ ion, which appears to generate more compact and organized regions by inducing the reticulation of the alginate phase.

The most substantial changes appear when considering the how many times the extrusion process was performed. For the samples F3-1X and F3-3X it becomes evident that the new perturbation creates enough turbulence to shift a structure composed by horizontally placed plates (F3-1X) to a system of pores of uniform size (F3-3X), which can relate to the incorporation of air inside the bioink. Regarding to the formulation F6, it is evident that, since the alginate phase is now ionically reticulated, it is harder to introduce enough turbulence to produce a structural shift through the extrusion process.

\section{Thermogravimetric Analysis}

Thermogravimetry is a technique that helps to obtain information about the humidity content or the thermal stability of a sample when exposed to inert or oxidizing environments, which can be useful in the characterization of materials with hydrophilic components. The results of this analysis are relevant since biomaterials must be sterilized before they can be used. This can be achieved by exposing the sample to high temperatures, which can generate decomposition or unwished chemical reactions, or to gamma or ultraviolet radiation, which can cause depolymerization and a decrease in viscosity due to a reduction in the average molecular weight (Ackert-Burr, 2010).

The results of this assay are shown as TGA and Differential Thermo Gravimetry (DTG) graphs in Figure 6. In general, every graph has from three to four peaks corresponding to decomposition or dehydration temperatures of the components of the bioinks: NaAlg, PNIPAm and $\mathrm{ZnSO}_{4}$ (Ingraham and Marier, 1967; Straszko et al., 1997; Jones et al., 2013). According 
to the literature, NaAlg suffers a three-stage decomposition: dehydration $\left(75-96^{\circ} \mathrm{C}\right)$, an initial decomposition to form $\mathrm{Na}_{2} \mathrm{CO}_{3}\left(200-240^{\circ} \mathrm{C}\right)$ and carbonization $\left(600-750^{\circ} \mathrm{C}\right)$ (Soares et al., 2004). Additionally, PNIPAm decomposes at temperatures above $330^{\circ} \mathrm{C}$ due to pyrolysis reactions (Bauri et al., 2013). All the peaks located under $255^{\circ} \mathrm{C}$ were attributed to the natural polymer and, more specifically, those located under $150^{\circ} \mathrm{C}$ were associated to dehydration. The peaks located in the range of $354-362{ }^{\circ} \mathrm{C}$ were associated to the decomposition of PNIPAm.

From the obtained results, it can be concluded that the bioinks can be thermally sterilized by using an inert component, such as $\mathrm{N}_{2}$ or Ar, as long as the temperature does not exceed $150^{\circ} \mathrm{C}$. For that effect, it is recommended to use an autoclave.

\section{Hemolysis Test}

This is one of the most important assays in the development of scaffolds and bioinks, since they are destined to constitute the extracellular matrix where the cells will proliferate, grow and differentiate to eventually generate tissues or organs. Bioinks must be atoxic, and this assay allows to quantitatively analyze the affinity level between cells and test agents at different concentrations so, in case any component is revealed as incompatible, the critic concentration can be determined to avoid any counterproductive effects regarding cell viability (Fosmire, 1990; Evans et al., 2013).

The hemoglobin that is released as a product of erythrocytes lysis can be detected through a spectroscope in a wide range of wavelengths. The hemolysis level of a sample at a given concentration can be compared with the lysis level of a negative (does not produce cell lysis) and a positive control (total cell obliteration) to determine how lethal it is. The surfactant, Triton $\mathrm{X}-100$, does not have any absorbance peaks in the range of 400$600 \mathrm{~nm}$, so it does not interfere with the normalization of the data (Evans et al., 2013). The selected analysis wavelength, $540 \mathrm{~nm}$, belongs to the visible electromagnetic radiation spectrum, so it can be perceived by the human eye. Because of this, the aqueous solution progressively turns red as the lysis level increments, as shown in Figure 10. The obtained results for this assay are shown in Figure 8.

For this test it was expected that the hemolysis level would increase proportionally to the concentration of the test agents, since the used solvent was PBS, an isotonic solution which a $\mathrm{pH}$ of 7.4. However, for most of the studied test agents, the higher hemolysis levels were obtained for the lowest concentration $(75 \mu \mathrm{g} / \mathrm{mL})$. Many of the discrepancies from the expected results were attributed to either the margin of error or experimental errors. In any case, if a test agent is non-hemolytic at high concentrations, this tendency should not change at lower ones.

According to the literature, hemolytic indexes of $0-2,2-5$, and 5-100\% can be interpreted as non-hemolytic, slightly hemolytic and hemolytic, respectively (ASTM International, 2017). So, it can be concluded that the developed formulations are nonhemolytic and could potentially be used as bioinks.

\section{CONCLUSIONS}

Bioprinting technologies are still on their early stages of development and there are plenty of researches left to do regarding many questions that remain unanswered. The designing process of bioinks should still be a priority, since it represents one of the main components of bioprinting. The use of gel-based formulations seems to be the right lead to follow in the extrusion-based technique. The results presented in this article represent an opportunity for further investigations so enhancements can be made in any of the multiple aspects of bioprinting since it has been seen in the literature many developments oriented to the most diversified human tissues and organs by applying bioinks.

Several assays were carried out to determine if six formulations based on NaAlg and PNIPAm could be used as bioinks in bioprinting.

It was determined through UV-Vis spectroscopy that they have a LCST close to the human body temperature due to the presence of PNIPAm. This thermosensitive behavior positions the developed formulations as smart gels.

The scanning electron microscopy analysis revealed that the extrusion process affects the distribution of pores due to the introduction of turbulence. The presence of $\mathrm{Zn}^{2+}$ helps to reduce the impact of the extrusion process due to the ionic reticulation of the alginate phase.

Only the formulations F5 and F6 have the required viscosity to be applied in extrusion-based bioprinting.

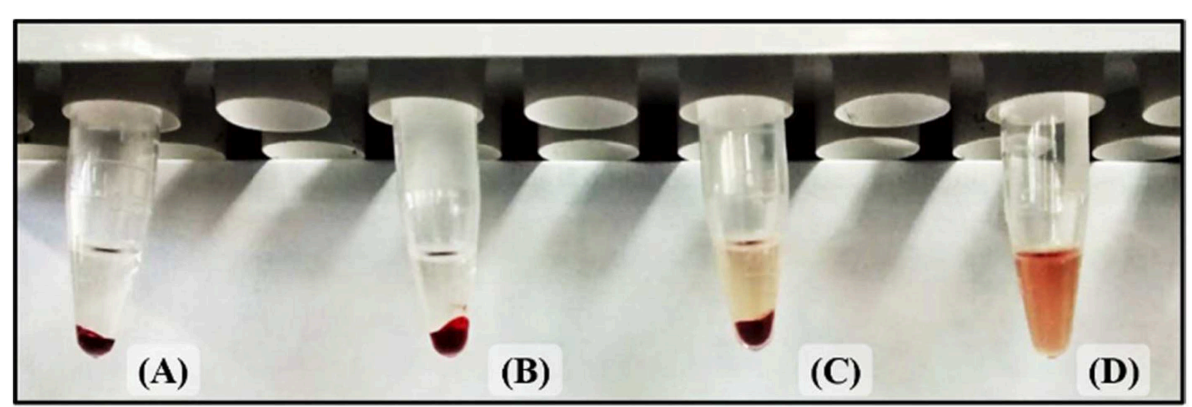

FIGURE 10 | Increase in hemoglobin concentration due to hemolysis. (A) Negative control (atoxic). (B) Low hemolysis level. (C) High hemolysis level. (D) Positive control (toxic). 
The TGA revealed that the designed gels can undergo thermally sterilization processes without any decomposition risk under $150^{\circ} \mathrm{C}$ and inert environments.

Furthermore, the hemolysis assay results positioned the formulations as hemocompatibles.

The formulations F5 and F6 could be used as smart bioinks for applications such as cell culture, cell and biomolecules microencapsulation, tissue engineering, and bioprinting.

\section{DATA AVAILABILITY}

All datasets generated for this study are included in the manuscript/supplementary files.

\section{AUTHOR CONTRIBUTIONS}

All the authors are partners of a long bilateral institutional partnership between Simón Bolívar University and Renato Archer Information Technology Center (CTI). The idea of this work borned in CTI (Brazil) when Professor MAS spent some months and brought PNIPA material and we

\section{REFERENCES}

Ackert-Burr, C. (2010). Low-temperature sterilization: are you know? Perioperative Nurs. Clin. 5, 281-290. doi: 10.1016/j.cpen.2010.05.002

ASTM International (2017). ASTM F756-17, Standard Practice for Assessment of Hemolytic Properties of Materials. West Conshohocken: ASTM International.

Atala, A., and Yoo, J. (2015). Essentials of 3D Biofabrication and Translation. Oxford: Elsevier.

Bauri, K., Roy, S., Arora, S., and De, P. (2013). Thermal degradation kinetics of thermoresponsive poly(N-isopropylacrylamide-co-N,Ndimethylacrylamide) copolymers prepared via RAFT polymerization. J. Therm. Anal. Calorim. 111, 753-760. doi: 10.1007/s10973-0122344-0

Bishop, E., Mostafa, S., Pakvasa, M., Luu, H., Lee, M., Wolf, J., et al. (2017). 3-D bioprinting technologies in tissue engineering and regenerative medicine: Current and future trends. Genes Dis. 4, 185-195. doi: 10.1016/j.gendis.2017.10.002

Carrero, M., Posada, J., and Sabino, M. (2018). Intelligent copolymers based on Poly-N-Isopropylacrilamide (PNIPAm) with potential use in biomedical applications, Part I: PNIPAm functionalization with 3butenoic acid and piperazine. Int. J. Adv. Med. Biotechnol. 1, 13-21. doi: 10.25061/2595-3931/IJAMB/2018.v1i1.9

Castro, E., Mosquera, V., and Katime, I. (2012). Dual drug release of triamterene and aminophylline from poly (N-Isopropylacrylamide) hydrogels. Nanomat. Nanotechnol. 2, 1-9. doi: 10.5772/50338

Cornelissen, D.-J., Faulker-Jones, A., and Shu, W. (2017). Current developments in 3D bioprinting for tissue engineering. Curr. Opin. Biomed. Eng. 2, 76-82. doi: 10.1016/j.cobme.2017.05.004

Coronado, R., Pekerar, S., Lorenzo, A., and Sabino, M. (2010). Characterization of thermo-sensitive hydrogels based on poly( $\mathrm{N}$-isopropylacrylamide)/hyaluronic acid. Polym. Bull. 67, 101-124. doi: 10.1007/s00289-0100407-6

Correa, M. Y., and Sabino, M. (2018). Chemical modification of alginate with L-Cysteine to extend its use in drug delivery systems. Cellulose Chem. Technol. 52, 559-567. doi: 10.1016/j.jddst.2019. 05.018

Derakhshanfar, S., Mbeleck, R., Xu, K., Zhang, X., Xhong, W., and Xing, M. (2018). 3D bioprinting for biomedical devices and tissue engineering: idealized the innovation of a smart bioink to be used in our test platform bioprinter. FM executed all the laboratory work presented in this paper under supervision of MS. RR followed remotely the work, provided subsidies information related to bioprinting and information technology aspects and contributed on the paper writing. JS worked as a consultant coauthor of the work and provided all the information technology infrastructure where the partnership has been run. MS advised his student. FM participated in all the experimental work and wrote the paper.

\section{FUNDING}

This work was supported by the CNPq through INCT-Regenera.

\section{ACKNOWLEDGMENTS}

The authors would like to thank the Laboratory E-USB Microscopy Section, also to the Polymer Laboratory in the Venezuelan Institute of Scientific Research (IVIC) and Laboratory CERTBIO-UFCG (Brazil) and the National Institute of Science and Technology on Regenerative Medicine (INCTRegenera) (Brazil). a review of recent trends and advances. Bioact. Mater. 3, 144-156. doi: 10.1016/j.bioactmat.2017.11.008

Dusseault, J., Tam, S., Ménard, M., Polizu, S., Jourdan, G., Yahia, L., et al. (2006). Evaluation of alginate purification methods: effect on polyphenol, endotoxin and protein contamination. J. Biomed. Mater. Res. A, 76, 243-251. doi: 10.1002/jbm.a.30541

El-Sherbiny, I., and Yacoub, M. (2013). Hydrogel scaffolds for tissue engineering: progress and challenges. Glob. Cardiol. Sci. Pract. 2013, 316-432. doi: $10.5339 /$ gcsp. 2013.38

Evans, B., Nelson, C., Yu, S., Beavers, K., Kim, A., Li, H., et al. (2013). Ex vivo red blood cell hemolysis assay for the evaluation of ph-responsive endosomolytic agents for cytosolic delivery of biomacromolecular drugs. J. Vis. Exp. 73, 1-5. doi: $10.3791 / 50166$

Fosmire, G. (1990). Zinc toxicity. Am. J. Clin. Nutr. 51, 225-227. doi: 10.1093/ajen/51.2.225

Gandhi, A., Paul, A., Oomen, S., and Kumar, K. (2015). Studies on thermoresponsive polymers: phase behaviour, drug delivery and biomedical applications. Asian J. Pharm. Sci. 10, 99-107. doi: 10.1016/j.ajps.2014.08.010

Groll, J., Burdick, J., Cho, D.-W., and Derby, B. (2018). A definition of bioinks and their distinction from biomaterial inks. Biofabrication 11:013001. doi: 10.1088/1758-5090/aaec52

Gungor-Ozkerim, P., Inci, I., Zhang, Y., and Dokmeci, M. (2018). Bioinks for 3D bioprinting: an overview. Biomater. Sci. 6, 915-946. doi: 10.1039/C7BM00765E

He, Y., Yang, F., Zhao, H., Gao, Q., Xia, B., and Fu, J. (2016). Research on the printability of hydrogels in 3D bioprinting. Sci. Rep. 6, 1-13. doi: 10.1038/srep29977

Ingraham, T., and Marier, P. (1967). Kinetics of the thermal decomposition of $\mathrm{ZnSO}_{4}$ and $\mathrm{ZnO}_{2} \mathrm{ZnSO}_{4}$. Can. Metall. Q. 6, 249-261. doi: $10.1179 / \mathrm{cmq} \cdot 1967.6 .3 .249$

Janna, W., and Simpson, M. (2008). "Newtonian and non-Newtonian fluids: velocity profiles, viscosity data, and laminar flow friction factor equations for flow in a circular duct," in Proceedings of IMECE 2008 (Boston, MA: ASME 2008 International Mechanical Engineering Congress and Exposition).

Jones, F., Tras, H., Lindberg, D., Zhao, L., and Hupa, M. (2013). Thermal stability of zinc compounds. Energy Fuels 27, 5663-5669. doi: 10.1021/ef400505u

Lemanowicz, M., Gierczycki, A., Kuznik, W., Sancewicz, R., and Imiela, P. (2014). Determination of lower critical solution temperature of thermosensitive flocculants. Miner. Eng. 69, 170-176. doi: 10.1016/j.mineng.2014.07.022 
Li, N., Fane, A., Winston, W., and Matsuura, T. (2008). Advanced Membrane Technology and Applications. New Jersey, NJ: John Wiley and Sons. doi: 10.1002/9780470276280

Li, Y.-C., Shrike Zhang, Y., Akpek, A., and Khademhosseini, A. (2017). $4 \mathrm{D}$ bioprinting: the next-generation technology for biofabrication enabled by stimuli-responsive materials. Biofabrication 9:012001. doi: 10.1088/1758-5090/9/1/012001

Markstedt, K., Mantas, A., Tournier, A., Martínez Ávila, H., Hägg, D., and Gatenholm, P. (2018). 3D bioprinting human chondrocytes with nanocellulosealginate bioink for cartilage tissue engineering applications. Biomacromolecules 16, 1489-1496. doi: 10.1021/acs.biomac.5b00188

Mironov, V., Kasyanov, V., Drake, C., and Markwald, R. (2008). Organ printing: promises and challenges. Regen. Med. 3, 93-103. doi: 10.2217/17460751.3.1.93

Nair, K., Ghandi, M., Khalil, S., Yan, K., Marcolongo, M., Barbee, K., et al. (2009). Characterization of cell viability during bioprinting processes. Biotechnol. J. 4, 1168-1177. doi: 10.1002/biot.200900004

Park, J., and Lakes, R. (2007). Biomaterials. An Introduction. New York, NY: Springer.

Pati, F., Gantelius, J., and Svahn, H. (2016). 3D bioprinting of tissue/organ models. Angew. Chem. Int. Ed. 55, 4650-4665. doi: 10.1002/anie.201505062

Raviv, D., Zhao, W., McKnelly, C., Papadopoulou, A., and Kadambi, A. (2014). Active printed materials for complex self-evolving deformations. Sci. Rep. 4:7422. doi: $10.1038 /$ srep07422

Rezende, R., Kasyanov, V., Mironov, V., and Lopes da Silva, J. (2015). Organ printing as an information technology. Proc. Eng. 110, 151-158. doi: 10.1016/j.proeng.2015.07.023

Sabino, M. A., Loaiza, M., Dernowsek, J., Rezende, R., and Da Silva, J. V. L. (2017). Técnicas para la fabricación de andamios poliméricos con aplicaciones en ingeniería de tejidos. Rev. LatinAm. Metal. Mat. 37, 1-27.
Soares, J., Santos, J., Chierice, G., and Cavalheiro, E. (2004). Thermal behavior of alginic acid and its sodium salt. Eclética Quím. 29, 53-63. doi: 10.1590/S0100-46702004000200009

Straszko, J., Olszak-Humienik, M., and Mozejko, K. (1997). Kinetic of thermal decomposition of ZnSO4.7H2O. Thermochim. Acta 292, 145-150. doi: 10.1016/S0040-6031(96)03114-0

Vieira, J., Posada, J., Rezende, A., and Sabino, M. (2014). Starch and chitosan oligosaccharides as interpenetrating phases in Poly(N-Isopropylacrylamide) injectable gels. Mater. Sci. Eng. 37, 20-27. doi: 10.1016/j.msec.2013. 12.005

Wang, M., He, J., Liu, Y., and Li, M. (2015). The trend towards in vivo bioprinting. Int. J. Bioprinting 1, 15-26. doi: 10.18063/IJB.2015. 01.001

Yin, J., Yan, M., Wang, Y., Fu, J., and Suo, H. (2018). 3D bioprinting of low-concentration cell-laden Gelatin Methacrylate (GelMA) bioinks with a two-step cross-linking strategy. Appl. Mater. Interfaces 10, 6849-6857. doi: 10.1021/acsami.7b16059

Conflict of Interest Statement: The authors declare that the research was conducted in the absence of any commercial or financial relationships that could be construed as a potential conflict of interest.

Copyright (C) 2019 Montero, Rezende, da Silva and Sabino. This is an open-access article distributed under the terms of the Creative Commons Attribution License (CC $B Y)$. The use, distribution or reproduction in other forums is permitted, provided the original author(s) and the copyright owner(s) are credited and that the original publication in this journal is cited, in accordance with accepted academic practice. No use, distribution or reproduction is permitted which does not comply with these terms. 